\title{
Hábito lector en estudiantes de primaria: influencia familiar y del plan lector del centro escolar
}

\author{
Reading Habit in Primary School Students: Influence of Family and \\ School Reading Plan
}

\author{
María del Mar Martínez Díaz \\ Ana Torres Soto \\ Universidad de Murcia
}

Recibido: 23/01/2019

Aceptado: 09/04/2019

\begin{abstract}
In Primary School reading competence is a fundamental part of linguistic communication competence. And it has also been determined that its development in the early stages of education favors the academic success of students. Considering these ideas, a pilot study limited to one educational centre was carried out, a transversal cut, using a survey of closed-ended questions, on the value of the family in the development of the reading habit, investigating and showing the implications of this in the development policy of the Reading Plan and analysing whether this implication influenced the academic results of the students. This survey was administered to a sample of 51 students in the third year of Primary school. The management of the centre and the coordinators of the section were also interviewed. Once the results were obtained, the data obtained corroborated that the reading habit of the students is associated above all with school tasks and not so much with the taste or pleasure of reading in leisure time. It has also been determined that the attitude of the family has a clear influence on the development of the reading habit. And, finally, it could be verified that the academic performance of the students was related to this habit. It concludes with an emphasis on the necessary training of teachers to encourage the students' reading habits and family involvement in the Reading Plan.
\end{abstract}

KEY WORDS: Reading habit; Primary School; Reading programmes; Family; academic performance.

\section{RESUMEN}

En Educación Primaria la competencia lectora es una parte fundamental de la competencia en comunicación lingüística. Se ha determinado que su desarrollo en las primeras etapas educativas favorece el éxito académico de los estudiantes. Considerando estas ideas, se realizó un estudio piloto limitado a un centro educativo, de corte transversal, empleando una encuesta de preguntas cerradas sobre el valor de la familia en el desarrollo del hábito lector, investigando y mostrando las implicaciones de ésta en la política de desarrollo del Plan Lector y analizando si esta implicación influía en los resultados académicos del alumnado. Dicha encuesta se administró a una muestra de 51 estudiantes de tercer curso de Primaria. También se entrevistó a la dirección del centro y a los coordinadores de tramo. Una vez obtenidos los resultados, los datos alcanzados corroboraron que el hábito lector de los estudiantes está asociado sobre todo a las tareas escolares y no tanto al gusto o al placer de leer en tiempo de ocio. También se ha determinado que la actitud de la familia posee una clara influencia en el desarrollo del hábito lector. Y, finalmente, se pudo comprobar que el rendimiento académico de los alumnos estaba relacionado con dicho hábito. Se concluye poniendo de relieve la necesaria formación y capacitación del profesorado para incentivar el hábito lector del alumnado y la implicación familiar en el Plan Lector del centro.

PALABRAS CLAVE: Hábito de lectura; Educación Primaria; Programa de lectura; Familia; Rendimiento académico.

Authors' correspondence address:

María del Mar Martínez Díaz, Universidad de Murcia.

Ana Torres Soto, Universidad de Murcia, Departamento de Didáctica y Organización Escolar, email: ana.t.s@um.es. ORCID: 0000-0003-0832-8580 


\section{Introducción}

La lectura ocupa un papel nuclear en los procesos de enseñanza y aprendizaje. Numerosos autores han señalado la importancia del desarrollo del hábito lector en la etapa primaria, como condicionante de la actitud de los estudiantes hacia el estudio y el aprendizaje en etapas posteriores (Artola, Sastre y Barraca, 2017), como determinante para la adquisición de conocimientos y el desarrollo del espíritu crítico (Esteban Peregrina, 2017) y como base del éxito académico, de sus oportunidades educativas, de trabajo e inserción social (Treviño y otros, 2007). Se considera, por tanto, que su carácter transversal conlleva efectos colaterales positivos o negativos en el alumnado, de manera que lograr o no un hábito lector adecuado, facilitará o dificultará la comprensión de los textos y, por consiguiente, los resultados y el éxito académico (Gutierrez-Braojos y Salmerón Pérez, 2012). Por su parte, Lluch y Sánchez-García (2017) consideran que su construcción es tarea compleja y supone gran esfuerzo y trabajo continuado. Todo ello determina la mirada escolar desde la que se aborda. Al configurarse como una competencia básica del currículo supone que todas las áreas o materias escolares han de favorecer su desarrollo y adquisición (Pérez Esteve, 2009). Se considera, asimismo, como una habilidad básica y necesaria, por su carácter instrumental, para abordar cualquier actividad escolar, dado que gran parte de la información es transmitida por escrito y es necesario comprenderla para interpretar su contenido (Salvador Mata, Gallego y Mieres, 2007). En este sentido, la lectura se hace imprescindible para acceder a los contenidos escolares.

Para conseguir desarrollar un hábito lector y un compromiso por la lectura se hace necesario trabajarla en diversos contextos. El papel desempeñado por las instituciones escolares y por las familias es transcendental y decisivo en la educación lectora del alumnado. La literatura recoge profusamente evidencias de la influencia familiar en el desarrollo del hábito lector (Beltrán, LópezEscribano y Rodríguez-Quintana, 2006; Blanch, Durán, Valdebenito y Flores, 2013; Fernández, García y Prieto, 1999; Galán y López-Jurado, 2016; Gil Flores, 2009; Mora-Figueroa, Topping, Dekhinet y Seedyk, 2011; Moreno, 2002) aludiendo a las múltiples variables que lo condicionan. Por su parte, los centros escolares incorporan políticas relacionadas con la elaboración de planes de lectura y biblioteca escolar en los centros, entendiéndose que ello constituye una oportunidad para impulsar el tratamiento didáctico de la lectura y la integración de acciones que se vienen realizando de manera aislada (Serna, Rodríguez y Etxaniz, 2017) y puntual.

El reconocimiento de la importancia e influencia de estos dos agentes -familia y escuela- en la educación lectora de los niños y niñas motivan el estudio que aquí se presenta. Específicamente, los objetivos perseguidos pretenden indagar en los gustos, preferencias y hábito lector de alumnos de tercer curso de Educación Primaria, incidir en la influencia de la familia en dicho hábito y conocer la importancia que se le otorga a este agente en el Plan Lector del centro educativo donde se ha llevado a cabo el estudio.

Para ello, será presentado un marco conceptual que fundamenta inscribir la competencia lectora en una concepción que va más allá de una perspectiva mecanicista (Solé, 2012), conjugando, asimismo, la importancia de la familia y la escuela en la construcción de la competencia lectora. Posteriormente, considerando las características metodológicas más destacables de la investigación, se exponen los resultados que darán respuesta a los objetivos e hipótesis planteadas en este estudio.

\section{Estado de la cuestión}

La competencia lectora es definida como "la capacidad de un individuo para comprender, utilizar y reflexionar sobre textos escritos, con el propósito de alcanzar sus objetivos personales, desarrollar su conocimiento y sus capacidades, y participar en la sociedad" (OCDE, 2016, p.7). Esta definición otorga una incuestionable importancia a la lectura en el proceso de enseñanza y aprendizaje, en la adquisición de las competencias básicas establecidas para Educación Primaria y, por consiguiente, en el desarrollo integral del alumnado. En el artículo 7 del Real Decreto 126/2014, de 28 de febrero, por el que se establece el currículo básico de la Educación Primaria, se establece que uno de los objetivos de la etapa es el de desarrollar hábitos de lectura y se determina que para fomentar el hábito lector se dedicará un tiempo diario a la misma. Para garantizar este objetivo, en el 
Decreto 198/2014, de 5 de septiembre, por el que se establece el currículo de la Educación Primaria en la Comunidad Autónoma de la Región de Murcia, se presentan dos instrumentos básicos: a) la asignatura Lectura Comprensiva a cursar por el alumnado durante los tres primeros cursos de la etapa; y b) la adopción de planes o programas específicos en los centros escolares que fomenten el hábito de la lectura.

Normativamente, el incentivo de políticas de fomento y promoción de la lectura en las instituciones escolares ha trazado el camino para promover el hábito y el gusto por la lectura entre el alumnado. Desde la legislación educativa se contempla, además, como un factor determinante en la adquisición de la competencia lingüística y como un factor indisociado de los aprendizajes curriculares y sociales que tendrán lugar a lo largo de la vida (Yubero y Larrañaga, 2010). Su aplicación a nivel institucional debe avanzar en proyectos coherentes que pongan en valor la lectura, el conocimiento y la cultura (Solé, 2012), mediante la articulación de recursos materiales y humanos, de formación y de asesoramiento del profesorado, que así lo aseguren. A nivel de aula, el trabajo del docente no se ha de basar únicamente en proporcionar libros y/o textos para desarrollar la competencia lectora, sino en invitar al alumnado a la reflexión, a la comprensión, contribuyendo así a la obtención de un aprendizaje significativo (Gaviro, 2010). Precisamente Solé (2012) argumenta que la lectura no debe concebirse solo como un medio para acceder al conocimiento, sino que permite (o debe permitir) dotar de estrategias que permitan pensar y aprender. De acuerdo con Gaviro (2010), para que los alumnos desarrollen su competencia lectora es necesario e imprescindible dedicarle tiempo a la lectura. Ello implica aprender haciendo, planteando al alumnado nuevos problemas que permitan desarrollar ese hábito (Perrenaud, 2004).

Desarrollar la competencia lectora supone, por tanto, entenderla desde una concepción amplia y compleja, vinculada a un proyecto personal que no se restringe únicamente a razones estrictamente instrumentales (ligadas a obligaciones escolares), sino que se orienta al desarrollo, el crecimiento y la inserción social (Solé, 2012). Supone, además, su reconstrucción y complejidad, a la vez que su enriquecimiento, cada vez que se utiliza para satisfacer propósitos sociales y personales, no solamente para requerimientos académicos. E implica, asimismo, la intencionalidad de comprender y formarse como lector crítico, de llegar a formas más profundas de lectura (Álvarez-Álvarez y Pascual-Díez, 2013). No obstante, si bien este proceso complejo de concepción de la competencia lectora se configurará a lo largo de la vida, el hábito lector es un proceso que ha de iniciarse desde los primeros años. El hábito lector se encuentra relacionado, de acuerdo con Zaragoza (2016), con el comportamiento, la actitud, la orientación positiva hacia la lectura; e incorpora algún tipo de satisfacción personal que refuerza dicho hábito (Yubero y Larrañaga, 2010). Además, señalan Aguilar, Aragón, Navarro, Delgado y Marchena (2017), que los mejores lectores tendrán ventajas en el desarrollo del vocabulario, que a su vez facilitará la comprensión de la lectura, y al volverse esta más eficiente, aumentará su hábito.

$\mathrm{Y}$ en este proceso también es un elemento fundamental el entorno familiar. Como se ha mencionado anteriormente, numerosos son los estudios que coinciden en focalizar la atención en el contexto familiar y concretarlo como elemento influyente en el hábito lector del niño. Indican MoraFigueroa, Galán y López-Jurado (2016), que el hábito lector viene propiciado y condicionado, en gran medida, por la motivación, pudiendo ser favorecida esta actitud en el contexto familiar. En consideración con esta variable señalan que algunos estudios han relacionado la motivación lectora con la implicación de las familias en este hábito; otros estudios la vinculan con el ambiente literario del hogar; y otros la condicionan a las acciones intencionadas realizadas por la familia para fomentar la lectura. A modo de explicación, Strommen y Mates (2004) expusieron que el hábito lector se observa más desarrollado en aquellos alumnos que encuentran en la lectura un componente elemental en su espacio de ocio y, al mismo tiempo, participan de esta afición y gusto por la lectura con algún miembro de su familia, a través de la interacción y la comunicación. En este sentido, se podría señalar como aspecto clave para favorecer el hábito lector las condiciones que se encuentran en el ámbito familiar.

Escuela y familia, por tanto, han de estrechar acciones que permitan favorecer el hábito lector. Si bien se ha puesto de manifiesto que ambas instituciones se sitúan como elemento clave en ese 
proceso de "enganche", la familia no está "obligada" a satisfacer su desarrollo, quedando frecuentemente relegado a las actuaciones de la escuela. Socializar a las familias en actitudes participativas y comprometidas y en la capacitación de la educación lectora de sus hijos podría incidir en el desarrollo de una concepción deseable de su responsabilidad como formadores de lectores. Como interpretan Villiger, Niggli, Wandeler, y Kutzelmann (2012), las actitudes positivas hacia la lectura mejoran cuando existe un apoyo familiar basado en actitudes constructivas y positivas sólidas. Además, se ha demostrado que la implicación familiar incide, también, positivamente en el aprendizaje y el rendimiento escolar de los estudiantes (Gil Flores, 2009; Mora-Figueroa, Galán y López-Jurado, 2016). En este sentido, el desarrollo y consolidación de proyectos institucionales de fomento de la lectura debieran incorporar estrategias que refuercen la participación de la familia. Una forma de hacerlo es integrando acciones educativas en el hogar enlazadas con las acciones desarrolladas en la escuela. Y dando un paso más, con la creación de auténticas comunidades de aprendizaje.

En base a estas evidencias se refuerza la idea de la importancia que juegan la institución escolar y el entorno familiar en el proceso de desarrollo del hábito lector, no como agentes independientes, sino como agentes que se han de complementar y que han de configurarse como ambientes ricos y estimulantes para favorecer el hábito lector, la competencia lectora y, en consecuencia, el éxito escolar. Pero, ¿realmente se implica a las familias en los planes y proyectos de fomento de lectura del centro? ¿Se promueve el compromiso de las familias? Son escasas las investigaciones que muestran una relación estrecha entre familia y escuela en esta temática. En su mayoría tratan de investigar cómo se promueve el hábito lector en el hogar sin incidir en la relación familia-escuela que tan importante papel juegan de manera complementaria.

Partiendo de lo expuesto, este estudio tiene como objetivo fundamental conocer el hábito lector de un grupo de estudiantes de tercer curso de Educación Primaria, así como valorar la implicación familiar en la adquisición de dicho hábito a través de las respuestas del alumnado y de las valoraciones de profesores y del equipo directivo implicados en el Plan Lector del centro. Se pretende, finalmente, conocer si el hábito lector del alumnado influye en el rendimiento académico. Concretamente, se tratará de dar respuesta a los siguientes objetivos específicos: 1). Conocer los gustos, preferencias y el hábito lector de los estudiantes; 2). Valorar la implicación familiar en la adquisición del hábito lector a partir de las valoraciones del alumnado; 3). Conocer qué implicación tienen las familias en el Plan Lector del centro educativo; y 4). Analizar si existe relación entre el hábito lector y la influencia familiar y el rendimiento académico de los escolares en el área de Lectura Comprensiva. En relación con estos objetivos se plantean las siguientes hipótesis: 1). El Plan Lector del centro no incorpora estrategias que promuevan la participación e implicación de las familias para favorecer el hábito lector de sus hijos; y 2). Existe una relación positiva entre el hábito lector y el rendimiento académico de lo escolares en el área de Lectura Comprensiva.

\section{Metodología}

Se realizó un estudio piloto limitado a un centro educativo. Se pretendía conocer así las principales tendencias o dimensiones tentativas que, en estudios posteriores, podrán incorporarse a un diseño definitivo más amplio. El estudio se ha realizado a un grupo heterogéneo de estudiantes en cuanto a niveles de rendimiento académico y motivación por la lectura, de un centro al que acuden estudiantes procedentes de un contexto socioeconómico donde no existen desigualdades significativas. De manera concreta, se decidió recoger información de estudiantes de tercero de Primaria por ser el último curso donde se imparte la asignatura Lectura Comprensiva.

Para ello, se ha adoptado un diseño no experimental con una metodología de tipo descriptivo. En cuanto a su dimensión temporal, se trata de un estudio transversal que recoge información de un grupo de estudiantes y profesores de un centro concreto de la Región de Murcia en un momento determinado. Con objeto de contrastar la información que se pretendía recoger, se hizo un uso combinado de métodos cualitativos y cuantitativos. 


\subsection{Participantes}

Para llevar a cabo este estudio se recogió información de dos colectivos: 1) alumnado de tercer curso (por ser el último curso en el que se imparte la asignatura "Lectura Comprensiva"); y 2) dirección del centro y coordinadores de tramo.

El proceso de selección de los participantes se realizó mediante una técnica de muestreo no probabilístico intencional por accesibilidad (Bisquerra, 2012). Estuvo conformada por 51 alumnos, 34 niños $(66.7 \%)$ y 17 niñas $(33.3 \%)$, que cursaban $3^{\circ}$ de Educación Primaria $(\mathrm{n}=51)$ en un centro privado concertado de la comarca de Cartagena, en la Región de Murcia (España). El grupo de alumnos tenía edades comprendidas entre 8 y 10 años.

Los profesores participantes en el estudio fueron: la directora pedagógica de Educación Primaria (dada su participación en el proceso de elaboración del Plan Lector del centro) y los coordinadores de primer y segundo tramo de la etapa (por su representación como nexo de unión entre el equipo directivo y el personal docente del centro objeto de investigación).

\subsection{Instrumentos de recogida de información}

Se han empleado dos instrumentos de recogida de información: uno de carácter cuantitativo, el cuestionario (dirigido al alumnado); y otro de carácter cualitativo, la entrevista (dirigido a la directora y coordinadores de tramo). El primero de ellos, compuesto de 15 preguntas cerradas, recoge información sobre dos variables: 1) gustos, preferencias y hábito lector del alumnado (de la pregunta 1 a la 4), y 2) implicación familiar en el hábito lector (de la pregunta 5 a la 15). Las cuestiones planteadas respecto de la primera variable fueron las siguientes: ¿te gusta leer?; ¿tienes libros de lectura en casa que no sean los de texto, ni los leídos en el colegio?; además de la media hora diaria de lectura incluida en los deberes, ¿dedicas más tiempo a leer?; ¿acostumbras a leer en tu tiempo libre? En referencia a la segunda variable las preguntas formuladas fueron las siguientes: cuando tenías menos edad, ¿te leían libros?; ¿en casa se habla de libros?; ¿te compran o regalan libros de lectura?; ¿tus padres te preguntan sobre lo que estás leyendo?; ¿hay en tu casa un espacio dedicado a los libros de lectura?; ¿algún miembro de tu familia lee en su tiempo libre?; ¿suelen comprar en tu casa libros?; ¿se leen en tu casa libros en soporte digital?; ¿'sueles acudir a alguna librería o biblioteca con tus padres o familiares para comprar, coger o intercambiar libros?; ¿intercambias libros de lectura con tus hermanos, amigos o con algún miembro de tu familia?. El cuestionario arrojó una validez interna de .73 según el alfa de Cronbach.

Todas las preguntas fueron formuladas para conocer la acción voluntaria de la lectura y el tiempo de ocio del alumnado (pues se pretendía valorar el hábito lector), obviando todo aspecto vinculado a la obligatoriedad que puede conllevar la lectura derivada del proceso de enseñanza y aprendizaje. Las opciones de respuesta se presentaron en una escala tipo Likert con cuatro opciones de respuesta: nunca, a veces, con frecuencia y siempre.

El segundo instrumento de recogida de información, la entrevista, estuvo configurada por siete preguntas abiertas y pretendía indagar sobre el funcionamiento del Plan Lector en el centro escolar y las medidas contempladas en el mismo. Específicamente, en el presente artículo, tomamos como objeto de estudio aquellas cuestiones que ofrecen información sobre la participación de las familias en el Plan Lector y sobre las medidas concretas implementadas en el aula (concretamente, en el curso objeto de estudio). Estas preguntas fueron las siguientes: especialmente, con los alumnos de tercer curso de Educación Primaria, ¿qué medidas concretas se toman respecto a este plan?; ¿Está implicada la familia en el Plan de Fomento de la Lectura? ¿De qué forma?; ¿Se observan resultados favorables en los alumnos desde que el Plan se lleva a cabo?; ¿Crees que el Plan de Fomento de la Lectura está sujeto a medidas de mejora? ¿Cuáles?

Para la validez de contenido del instrumento se desarrolló un proceso de valoración de interjueces. El panel de expertos estuvo compuesto por 5 profesores, de los cuales 3 pertenecían al departamento de Didáctica y Organización Escolar de la Universidad de Murcia, y 2 al departamento de Didáctica de la Lengua y la Literatura de ISEN, Centro Universitario. Se utilizó una plantilla que incluía los siguientes criterios para valorar cada pregunta: adecuación, pertinencia y claridad, empleando una escala de 1 a 4 y una casilla por ítem para las propuestas de mejora. Estos expertos 
sugirieron modificaciones en cuanto al orden de preguntas para poder ofrecer una lógica en su estructura y la introducción de algunos matices en las cuestiones que garantizaron su fiabilidad y objetividad, estableciendo así una validez a los instrumentos y al estudio de dicha investigación.

\subsection{Procedimiento}

Se expuso a la dirección del centro educativo los objetivos de la investigación y se solicitó su colaboración. Seguidamente se solicitó el consentimiento informado de todos los posibles participantes, pidiendo autorización a los padres o tutores legales. Firmada dicha autorización e identificados los estudiantes, se realizó la administración de la prueba de manera colectiva en el aula. Posteriormente, se entrevistó a la directora del centro y a los coordinadores de tramo sobre el Plan Lector, y se preguntó al profesorado de la asignatura Lectura Comprensiva las calificaciones de los estudiantes en dicha asignatura con objeto de realizar las correlaciones pertinentes.

\subsection{Análisis de datos}

$\mathrm{El}$ análisis de los datos derivados del cuestionario fue realizado con el programa estadístico SPSS v24. Se utilizaron técnicas descriptivas (frecuencias, porcentajes...). Para cuantificar el hábito lector se creó un factor. Así para variables cuantitativas se utilizaron correlaciones bivariadas a través del coeficiente de correlación de Pearson y con el tamaño del efecto de Cohen.

\section{Resultados}

La presentación de los resultados será coherente con los objetivos específicos propuestos. En primer lugar, se presentan las respuestas obtenidas respecto a la valoración del alumnado sobre gustos, preferencias y hábito lector (4 primeros ítems) y sobre la implicación familiar en el hábito lector (11 últimos ítems). Seguidamente, se analiza la relación entre el hábito lector y el rendimiento académico, a partir de la correlación de Pearson. Finalmente, se presentan las valoraciones de la directora y los coordinadores de tramo en relación con la implicación familiar en el Plan de Fomento de la Lectura del centro.

\subsection{Gustos, preferencias y el hábito lector de los alumnos}

En relación con esta variable, se observa que un porcentaje elevado de alumnos (37.3) indicó que solo a veces les gusta leer (véase Figura 1); no obstante, porcentajes menos elevados, pero no muy distantes, dan muestra de que les gusta leer con frecuencia (31.4\%) o siempre (25.5\%).

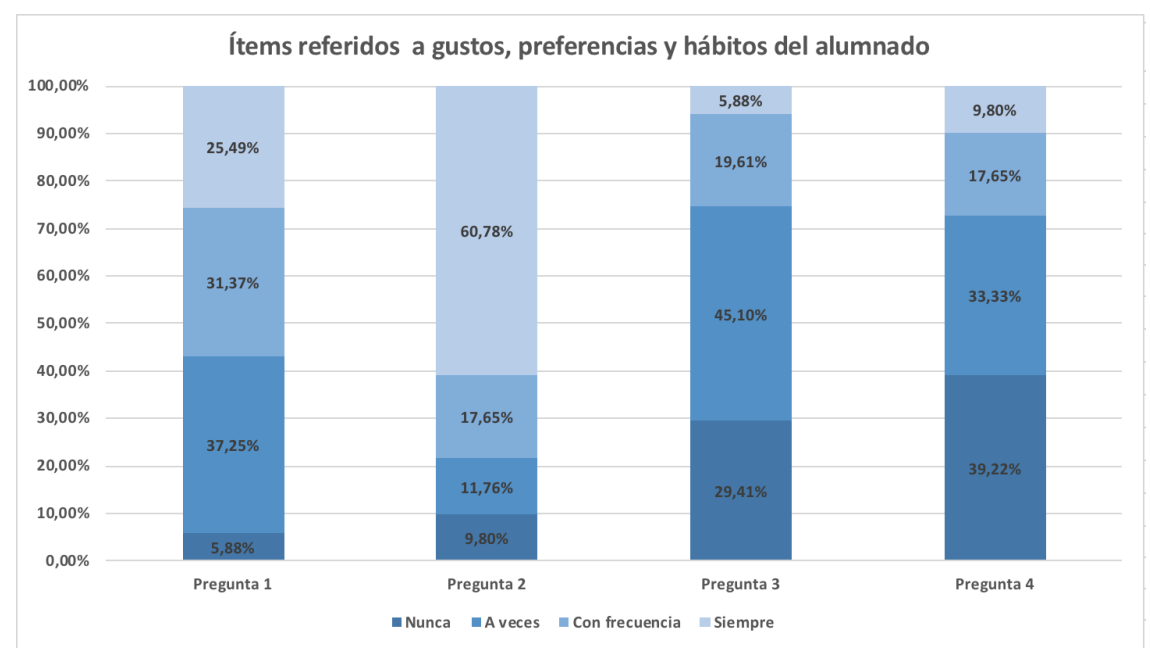

Figura 1. Gustos, preferencias y el bábito lector de los alumnos 
En contraposición con estos datos y haciendo referencia a la lectura diaria, un $45 \%$ de los alumnos (específicamente 23) contestó que solo a veces dedica más tiempo a leer además de la lectura escolar obligada, dato que confirma que casi la mitad de la muestra no concibe la lectura como un entretenimiento sino como una obligación escolar. Este resultado puede verificarse con los resultados obtenidos en la pregunta 4 , donde un $39.2 \%$ del alumnado ratifica que no acostumbran a leer en su tiempo libre. A pesar de ello, un número considerable de alumnos (concretamente 30) asegura que posee libros de lectura en casa, correspondiéndose con un $60.8 \%$ del total de la muestra.

\subsection{Implicación familiar en la adquisición del hábito lector}

En relación con la segunda variable, un $41.2 \%$ del alumnado declara que actualmente o cuando tenían menos edad sus familiares no les leían libros de lectura o cualquier otro tipo de texto. Un dato importante obtenido a través de este estudio, es el referente a la pregunta "en casa, ¿se habla de libros?", donde se puede comprobar que en el ámbito familiar tan solo a veces se habla de libros con un $47.1 \%$, quedando el ítem nunca bastante ajustado a este último con un $41.2 \%$. Otro dato a destacar es el obtenido en la pregunta "¿te compran o regalan libros de lectura?", donde solo a veces los alumnos reciben un libro como regalo, alcanzando el $39.2 \%$ de la muestra.

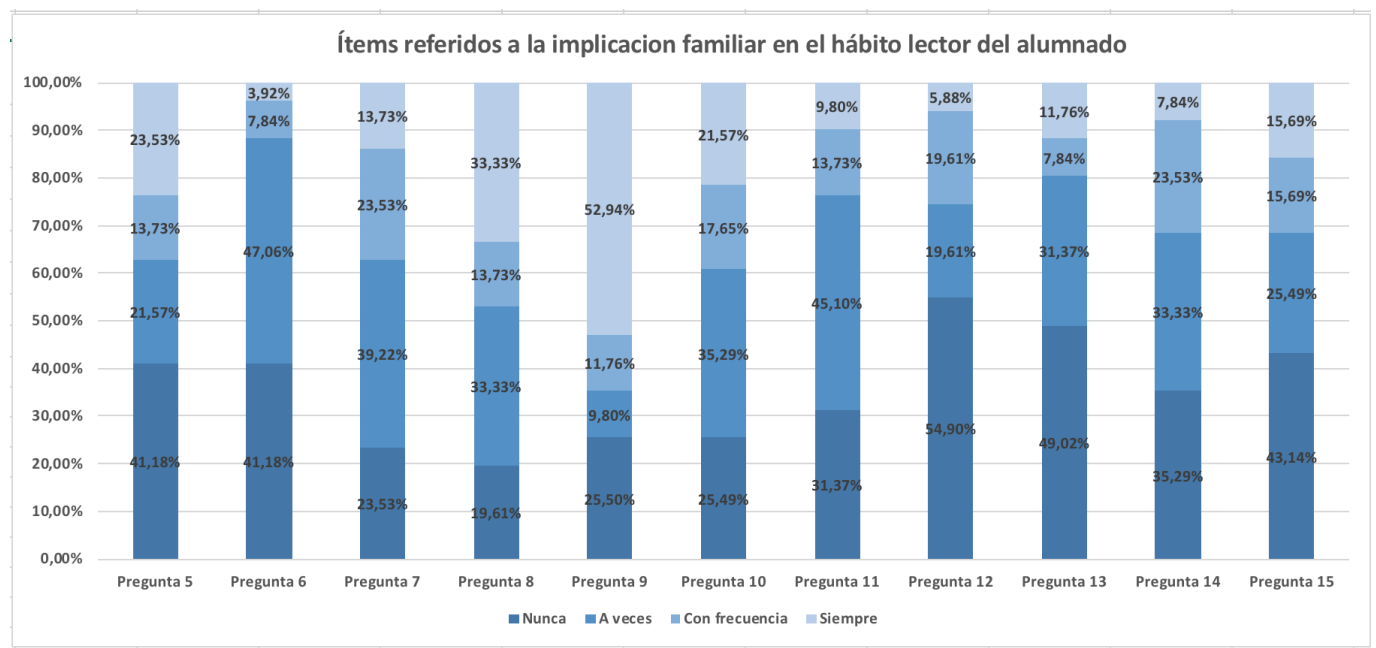

Figura 2. Implicación familiar en la adquisición del bábito lector

Observando los resultados podemos interpretar que los padres sí tienen cierto interés en la lectura de sus hijos puesto que 17 alumnos respondieron a partes iguales a veces y siempre, alcanzando un $33.3 \%$ respectivamente, por lo que interpretamos que en el ámbito familiar se interesan por sus lecturas en algún momento determinado. Una parte significativa del alumnado respondió que sí tienen un espacio dedicado a los libros de lectura, siendo la frecuencia a esta pregunta siempre $(52.9 \%)$. Por otro lado, y en conformidad con las preguntas 10 y 11, "¿algún miembro de tu familia lee en su tiempo libre?" y “isuelen comprar libros de lectura en casa?", el ítem predominante y por lo tanto la frecuencia obtenida es a veces (con 18 y 23 alumnos), y el porcentaje alcanzado un $35.3 \%$ y un $45.1 \%$, respectivamente.

Para finalizar este análisis, un elevado número de alumnos ha contestado que en casa nunca compran periódicos o revistas (54.9\%), no leen libros en soporte digital (49.0\%), no acuden a una librería o biblioteca (35.3) y no intercambian libros de lectura con familiares o amigos de su entorno (43.1\%).

4.3. Relación entre el hábito lector y el rendimiento académico en el área de Lectura Comprensiva

Respecto a la relación existente entre el hábito lector y el rendimiento académico de los estudiantes en el área de Lectura comprensiva, se observa una correlación positiva estadísticamente significativa, con tamaño del efecto medio bajo, entre el hábito lector y el rendimiento académico $(r=$ 
$.300 ; p=.033 ; \mathrm{n}=51)$. Esto supone que conforme aumentaba el hábito lector, aumentaba el rendimiento; lo que confirma la hipótesis de que existe una relación positiva entre el hábito lector y el rendimiento académico de lo escolares en el área de Lectura Comprensiva.

Tabla 1

Correlaciones entre las variables calificación y hábito lector en el total de la muestra

\begin{tabular}{llll}
\hline & & Calificación & Hábito Lector \\
\hline \multirow{2}{*}{ Calificación } & Correlación de Pearson & 1 &, $300^{*}$ \\
\cline { 2 - 4 } & \multicolumn{2}{c}{ Sig. (bilateral) } &, 033 \\
\cline { 2 - 3 } & $\mathrm{N}$ & 51 & 51 \\
\hline Hábito Lector & Correlación de Pearson &, $300^{*}$ & 1 \\
\cline { 2 - 3 } & Sig. (bilateral) &, 033 & \\
\cline { 2 - 3 } & $\mathrm{N}$ & 51 & 51 \\
\hline
\end{tabular}

4.4. Valoraciones de la directora y los coordinadores de tramo en relación con la implicación familiar en el Plan de Fomento de la Lectura del centro

De acuerdo con las respuestas de los participantes en las entrevistas, el Plan de Fomento de la Lectura del centro educativo incorpora una serie de medidas que tratan de incentivar la lectura tanto en el centro escolar como en casa. Las medidas que se contemplan para ser desarrolladas en el centro escolar son, de acuerdo con los coordinadores de tramo:

"la lectura diaria, la asignación de horas de apoyo, la lectura digital, la lectura en clase de producciones propias, la lectura mecánica individual y la planificación de la lectura". (CT1).

Indican que, gracias a este Plan, el alumnado realiza una lectura diaria en casa de treinta minutos, actividades de lectura comprensiva y lectura por turnos. Por tanto, la finalidad es la siguiente:

"que los alumnos conozcan y utilicen con corrección la lengua castellana oralmente y por escrito, como lengua de comunicación y aprendizaje, y desarrollen el hábito de la lectura como medio de enriquecimiento personal". (CT2)

Cuando se pregunta a los entrevistados por la implicación de las familias en el Plan indican que el papel de la familia en el proceso de desarrollo lector del alumnado consiste en el control de la media hora de lectura diaria, la cual forma parte de los deberes diarios de los alumnos, tal y como se ha mencionado con anterioridad. Los entrevistados confirman que para los estudiantes de $3^{\circ}$ de Educación Primaria (participantes de la investigación), el Plan de Fomento de la Lectura ejecuta unas medidas concretas que consisten en la lectura de tres libros durante el año académico en el aula, lectura diaria, realización de fichas de lectura, la concesión de un premio a final de curso al mejor lector tomando como referencia el carné por puntos y el préstamo de libros.

Una de las preguntas formuladas gira en torno a las propuestas o medidas de mejora que pueden considerarse en torno al Plan de Fomento a la Lectura. Ante esta cuestión hubo unanimidad en sus respuestas, declarando que:

"aunque el plan es muy completo en cuanto a programación y desarrollo, siempre está sujeto a mejoras como las que se pueden dar en lo relativo a la planificación y la calidad de las actividades programadas". (CT2)

"por supuesto, todo es mejorable. Cada año se revisa y se intenta completar innovando para hacerlo más atractivo a los alumnos y así mejorar los resultados". (CT1)

"sí, enriquecimiento de la biblioteca del aula, encuentros de autor, representaciones teatrales". 
Como podemos comprobar, señalan algunas propuestas de mejora concretas pero ninguno de los entrevistados menciona el ámbito familiar dentro de estas medidas, tan solo se marca la mejora en la calidad de las actividades ya planteadas o realizar otras acciones más atractivas y/o innovadoras. En este sentido, podemos afirmar que se prueba la hipótesis de que el Plan Lector del centro no incorpora estrategias que promuevan la participación e implicación de las familias para favorecer el hábito lector de sus hijos.

\section{Discusión y conclusiones}

Cabe resaltar que los datos obtenidos en esta investigación se han de tomar con cautela, especialmente porque son el resultado de un estudio piloto que pretende ser extendido (considerando y corrigiendo sus limitaciones) a una muestra mayor. En este sentido, se realizan afirmaciones de carácter especulativo y se informa sobre las principales tendencias y su correspondencia con otros estudios.

Como se indicaba en la introducción de este artículo, gracias a la adquisición de hábitos de lectura adecuados el alumnado desarrolla aspectos importantes como la comprensión o la actitud crítica, considerados como un elemento fundamental no solo en Lengua sino en el tratamiento de los contenidos de todas las áreas en general y para su participación activa en la sociedad (Artola, Sastre y Barraca, 2017; Esteban Peregrina, 2017; Salvador Mata, Gallego y Mieres, 2007; Treviño y et al., 2007). Por ello es importante tener en cuenta el valor de la lectura en la etapa de Educación Primaria y dar importancia a la adquisición del hábito, al gusto por la lectura y a la comprensión lectora, dado que resulta tan importante "leer para aprender" como "aprender a leer".

Los resultados confirman que gran parte del alumnado de tercer curso de Primaria no concibe la lectura como un hábito que forme parte de su día a día, al menos como hábito en su tiempo de ocio. Sí se observa, por el contrario, que esos momentos de lectura se elevan dada la obligatoriedad de la lectura escolar diaria, tal y como se ponía de manifiesto en el estudio de Yubero y Larrañaga (2010).

Las investigaciones han corroborado que la conducta familiar puede interferir en la adquisición de hábitos lectores y, en consecuencia, en el resultado de los aprendizajes del alumnado (Mayorga y Madrid, 2014; Mora-Figueroa, Galán y López-Jurado, 2016; Strommen y Mates, 2004). En este estudio se contempla que la influencia familiar en este proceso podría estar más asentada, pues aunque los resultados evidencian cierto interés de las familias por la lectura de sus hijos e hijas, adolece también un hábito poco estable en ellos (al menos, desde la percepción de los discentes). Esta evidencia muestra correspondencia con las afirmaciones de Mayorga y Madrid (2014), quienes interpretan que en muchas ocasiones las familias son conscientes de la importancia de la lectura para sus hijos e hijas, pero desconocen cómo desarrollar ese hábito y, consecuentemente, se produce una falta de implicación en dicho aprendizaje.

En relación con el ámbito familiar, la investigación refleja, asimismo, que el centro escolar lleva a cabo un Plan de Lectura basado en el trabajo independiente del alumno, que no involucra o implica directamente a las familias (salvo el control diario de la media hora de lectura). Del mismo modo, se ha observado que desde el centro no perciben la importancia de las familias en la adquisición de dicho hábito (al menos no se ha verbalizado explícitamente), por lo que determinamos que uno de los objetivos primordiales del centro debería comenzar por tomar consciencia de esta influencia, y continuar por motivar e incorporar el ámbito familiar en este proceso lector (más allá del control diario de la lectura). En este sentido, tal y como apuntamos en el marco teórico de este artículo, los centros escolares debieran obtener una serie de instrumentos suficientes y adecuados que les permitieran incentivar la participación de las familias a través de actividades motivadoras, cuya finalidad sea clara y con vistas a unos resultados a corto/medio plazo. Es importante que la escuela establezca mecanismos y estrategias de comunicación y capacitación de las familias que vaya más allá de un mero control del tiempo de lectura. Las actitudes de padres y madres hacia la lectura suelen ser 
imitadas por los hijos (Villiger, Niggli, Wandeler, y Kutzelmann, 2012) y el mero control no implica que haya actitudes positivas hacia la lectura por parte de las familias.

Respecto al rendimiento escolar y su asociación directa con el hábito lector a la que ya han apuntado numerosos autores (Artola, Sastre y Barraca, 2017; Esteban Peregrina, 2017; Gil Flores, 2009; Molina, 2006; Treviño y et al., 2007), la relación que se ha encontrado en este estudio (aunque con sus limitaciones determinadas por ser una muestra pequeña), ponen de relevancia la necesidad de intervenir más directamente en el hábito lector del alumnado, dada la confirmación de la existencia de una relación directa entre dicho hábito y los resultados escolares. Por todo ello, es imprescindible insistir en la importancia de desarrollar buenos hábitos asociados a la lectura no obligada tanto en la familia como en la escuela, dotando de estrategias y herramientas a ambos agentes que permitan mejorar no solo el hábito sino también, y en consecuencia, el aprendizaje futuro de los estudiantes. Como bien indica Granado (2013), "de poco sirven las políticas institucionales de fomento de la lectura y de las bibliotecas escolares sin un profesorado sólidamente formado para ello" (p.112), cuando se ha corroborado que hay una serie de carencias formativas en los maestros y maestras que derivan en escaso uso de las bibliotecas escolares y un uso generalizado del libro de texto.

En definitiva, es necesario que el profesorado esté altamente formado y capacitado para afrontar con éxito el reto de la educación lectora de sus estudiantes, con objeto de optimizar su labor en lo que respecta al desarrollo de la competencia, facilitar el hábito lector y contribuir así a incrementar su rendimiento académico. Además, sería conveniente tomar medidas para afianzar la relación con las familias y favorecer una participación más activa en el desarrollo de programas de tal envergadura como es el Plan Lector.

Para terminar, resaltamos la posibilidad de que, una vez ampliada la muestra, se desarrollen a nivel teórico y práctico la aplicabilidad de medidas y estrategias que permitan a centros y familias una mayor cohesión e implicación en el desarrollo de buenos hábitos de lectura.

\section{Referencias bibliográficas}

Aguilar, M., Aragón, E., Navarro, J.I., Delgado, C. y Marchena, E. (2017). Análisis del efecto san Mateo en un estudio longitudinal sobre el desarrollo lector durante la educación primaria $(1 . \circ \mathrm{a}$ 5.॰). European Journal of Education and Psychology, 10(1), 23-32. doi: 10.1016/j.ejeps.2016.07.001

Artola, T., Sastre, S. y Barraca, J. (2017). Diferencias de género en actitudes e intereses lectores: una investigación con alumnos españoles de primaria. Bordón, 69(1), 11-26. doi: 10.13042/Bordon.2016.37925.

Ahmed, M. D. (2011). Importancia de la lectura en infantil y primaria. Revista digital innovación y experiencias educativas, 38, 1-9.

Aristizábal, C. P. D., Rayo, K. D. O., Gómez, E. C. S., y Velázquez, F. A. B. (2012). La lectura como valor para la construcción del lector competente. Infancias Imágenes, 11(1), 107-113.

Beltrán, J., López-Escribano, C. y Rodríguez-Quintana, E. (2006). Precursores tempranos de la lectura. En C. Hernández y M. Veyrat (Coords.), Lingüística clinica y neuropsicología cognitiva (pp. 18 26). Madrid: UCM.

Blanch, S., Durán, D., Valdebenito, V. y Flores, M. (2013). The effects and characteristics of family involvement on a peer tutoring programme to improve the reading comprehension competence. European Journal of Psychology of Education, 28, 101-119. doi: 10.1007/s10212-012-0104-y

Decreto 198/2014, de 5 de septiembre por el que se establece el currículo de la Educación Primaria en la Comunidad Autónoma de la Región de Murcia. Boletín Oficial de la Región de Murcia, 206, 6 de septiembre de 2014, 33054- 33556.

Duque, C.P. y Vera, A.V. (2010). Exploración de la comprensión inferencial de textos narrativos en niños de preescolar. Revista colombiana de psicología, 19(1), 21-35.

Esteban Peregrina, A. (2017). Estado actual de la comprensión lectora en Educación Primaria. Revista Fuentes, 19(1), 15-37. doi: 10.12795/revistafuentes.2017.19.1.01 
Fernández, V., García, M. y Prieto, J. (1999). Los hábitos de lectura en España: características sociales, educativas y ambientales. Revista de Educación, 320, 379-390.

Gaviro, A. B. (2010). Procesos y factores que intervienen en la comprensión lectora en lengua materna (L1) y lengua extranjera (LE). Revista Autodidacta, 1(1), 87-99.

Gil Flores, J. (2009). Hábitos y actitudes de las familias hacia la lectura y competencias básicas del alumnado. Revista de Educación, 350, 301-322.

Gimeno, M. J. C. (2014). La lectura al amparo de la LOMCE: el Plan Lector. Forum Aragón: revista digital de FEAE-Aragón sobre organización y gestión educativa, 12, 37-41.

Granado. C. (2013). La formación inicial de maestros y maestras en educación lectora: perspectiva del alumnado y contenidos ofrecidos en tres universidades andaluzas. Investigación en la escuela, 80, 103-115. Disponible en: https://bit.ly/2YmlutO.

Gutiérrez-Braojos, C., y Salmerón Pérez, H. (2012). Estrategias de comprensión lectora: Enseñanza y evaluación en educación primaria. Profesorado, Revista de currículum y formación del profesorado, 16(1), 183-202.

Ley Orgánica 8/2013, de 9 de diciembre, para la mejora de la calidad educativa. Boletín Oficial del Estado, 295, de 10 de diciembre de 2013, 97858-97921.

Lluch, G. y Sánchez-García, S. (2017). La promoción de la lectura: un análisis crítico de los artículos de investigación. Revista Española de Documentación Científica, 40(4), e192. DOI: 10.3989/redc.2017.4.1450

Mayorga, M. J. y Madrid, D. (2014). El lector no nace, se hace: implicaciones desde la familia. Rivista Italiana di Educazione Familiare, (1), 81-88. doi: 10.13128/RIEF-14798.

Molina, L. (2006). Lectura y educación: los hábitos lectores y su repercusión académica en la ESO. Ocnos: Revista de estudios sobre lectura, 2, 103-120. Doi: 10.18239/ocnos 2006.02.07.

Mora-Figueroa, J., Galán, A. y López-Jurado, M. (2016). Efectos de la implicación familiar en estudiantes con riesgo de dificultad lectora. Ocnos: Revista de estudios sobre lectura, 15(1), 7-21. doi: 10.18239/ocnos 2016.15.1.866.

Mora-Figueroa, J., Galán, A. y López-Jurado, M. (2016). Eficacia de un programa de implicación familiar en la lectura de alumnado de 1. ${ }^{\circ}$ de Educación Primaria. Revista de Psicodidáctica, 21 (2), 375391. doi: $10.1387 /$ RevPsicodidact.14889

Moreno, E. (2002). La familia como institución sociocultural: su papel en la adquisición de los hábitos lectores. Portularia. Revista de Trabajo Social, 2, 309-324.

OCDE (2016). El programa PISA de la OCDE. Qué es y para qué sirve. París: Organización para la Cooperación y el Desarrollo Económicos. Recuperado de https://bit.ly/2dKyN0D.

Orden 20 de noviembre de 2014, de la Consejería de Educación, Cultura y Universidades por la que se regula la organización y la evaluación en la Educación Primaria en la Comunidad Autónoma de la Región de Murcia, Boletín oficial de la Región de Murcia, 270, de 22 de noviembre de 2014, 4259842677.

Pérez Esteve, P. (2009). La competencia lectora y la competencia en comunicación lingüística en el nuevo marco curricular: algunas claves para su desarrollo. Educatio Siglo XXI, 27(1), 13-32.

Perrenoud, P. (2004). Diez nuevas competencias para enseñar. Barcelona: Graó.

Pujolàs, P. (2008). El aprendizaje cooperativo como recurso y como contenido. Aula de innovación educativa, 170, 37-41.

Real Decreto 126/2014, de 28 de febrero por el que se establece el currículo básico de la Educación Primaria. Boletin Oficial del Estado, 52, 1 de marzo de 2014, 19349-19420.

Serna, M., Rodríguez, A. y Etxaniz, X. (2017). Biblioteca escolar y hábitos lectores en los escolares de Educación Primaria. Ocnos, 16(1), 18-49. doi: 10.18239/ocnos 2017.16.1.1205.

Simons, H. (2011). El estudio de caso: Teoría y práctica. Madrid: Ediciones Morata.

Solé, I. (2012). Competencia lectora y aprendizaje. Revista Iberoamericana de Educación, 29, 43-61.

Treviño, E., Pedroza, H., Pérez, G., Ramírez, P., Ramos, G. y Treviño, G. (2007). Prácticas docentes para el desarrollo de la comprensión lectora en primaria. México: Instituto Nacional para la Evaluación de la Educación. 
Topping, K., Dekhinet, R. y Zeedyk, S. (2011). Hindrances for parents in enhancing child language. Educational Psychology Review, 23, 413-455. doi: 10.1007/s10648-011-9169-4.

Yubero, S. y Larrañaga, E. (2010). El valor de la lectura en relación con el comportamiento lector. Un estudio sobre los hábitos lectores y el estilo de vida en niños. Ocnos: Revista de estudios sobre lectura, 6, 7-20. Doi: 10.18239/ocnos 2010.06.01.

Villiger, C., Niggli, A., Wandeler, C., y Kutzelmann, S. (2012). Does family make a difference? Midterm effects of a school/home-based intervention program to enhance reading motivation. Learning and Instruction, 22, 79-91. Doi: 10.1016/j.learninstruc.2011.07.001.

Zaragoza, F. (2016). El fomento de la lectura y la comprensión lectora. En E. Jiménez Pérez (coord.), La comprensión y la competencia lectoras (pp. 97-108). Madrid: Síntesis.

\section{Para citar este artículo}

Martínez Díaz, M.M. \& Torres Soto, A. (2019). Hábito lector en estudiantes de Primaria: influencia familiar y del Plan Lector del centro escolar. Revista Fuentes, 21(1), 103-114. [Fecha de consulta: dd/mm/aa]. doi: 10.12795/revistafuentes.2019.v21.i1.07. 